\title{
A CASE OF BRAIN ABSCESS
}

\author{
Lt Col PL PRASAD ${ }^{*}$, Col CG WILSON (Retd) ${ }^{+}$, \\ Lt Col K NAGENDRA ${ }^{\#}$, Col VIPAN CHANDAR ${ }^{* *}$
}

MJAFI 2002; $58: 175-176$

\section{Introduction}

I ntracranial infections still constitute a significant neurosurgical problem. The classical triad of headache, focal neurological deficit and fever is found only in $25 \%$ cases [1]. Diagnostic techniques like CT and MRI result in early and efficient diagnosis of brain abscess. Antibiotics have revolutionized the management of brain abscess and early institution of specific treatment has reduced mortality as well as sequelae.

\section{Case Report}

8 year old boy was admitted with complaints of low-grade intermittent fever, without chills and rigor of 5 days duration and excessive but easily arousable sleepiness of 2 days duration. A practitioner prior to admission treated him with cloroquine and ciprofloxacin. Examination revealed an average huilt boy weighing $21 \mathrm{Kg}\left(<5^{\text {th }} \%\right.$ ile $)$, drowsy, with pulse $70 /$ minute, respiration $30 /$ minute, temp $98^{\circ} \mathrm{F}$ and $\mathrm{BP} 100 / 60 \mathrm{~mm} \mathrm{Hg}$ right arm supine phase IV. There was no pallor, icterus, rash, cyanusis, and lymphadenopathy. There was no neurological deficit: pupils were of normal size and reacting to light. Fundus was normal. Other systems were essentially within normal limit. Haematological parameters were essentially normal. LFT, blood urea and serum creatinine values were within normal range. He was being treated symptomatically. Lumbar puncture (LP) was done which wals found to be under intense pressure and only few drops were collected which showed lymphocytes as the predominant cells. Other tests could not be done. However, within six hours of admission. the child developed bradycardia and hypertension of $120 / 90 \mathrm{~mm}$ of $\mathrm{Hg}$. Urgent CT scan showed hypo dense ring enhancing lesion in the left temporo parictal region measuring $3 \times 4.5 \mathrm{~cm}$ in size, suggesting cerebral abscess with significant mass effects (Fig-1). Child was then treated with Inj Sodium Penicillin 10 lac IV 4 hourly, Inj Gentamycin $50 \mathrm{mg}$ IV 12 hourly and Inj Chloromycetin $150 \mathrm{mg}$ IV 8 hourly (Inj Cefotaxime and Inj Amikacin could not be afforded by the patient). Child was also continued on Inj Dexamethasone and Inj Sodium Dilantin. Abscess was drained with twist drill method and $20-\mathrm{ml}$ thick brown pus was aspirated. Repeat aspirations were dry. The pus was sterile. Antibiotics were continued for 4 weeks. Child improved gradually within 48 hours and was later subjected to detailed ENT. dental and cardiovascular checkup. Dental caries was found without odontogenous cyst. No septic focus from otologic or cardiovascular source was detected

\section{Discussion}

Sir William Macewen of Glasgow performed surgery on 19 patients of brain abscess out of whom 18 recovered [3]. Amongst 384 ciases of brain abscess treated at All India Institute of Medical Sciences (AIIMS), New Delhi, 472 at Post Graduate Institute of Medical Sciences (PGI) Chandigarh and 480 at Medical College Trivandrum, nearly 40\% were reported to be otogenic and etiology could be established in $15.8 \%$ cases $[4]$. Brain abscess can also occur spontaneously [5].

The presenting symptoms and neurological features in brain abscess depend upon factors like location. size, number, virulence of organisms, degree of cerebral edema and host response to infection. Intractable headache. unresponsive to therapy due to rapidly expanding intra-cranial mass lesion, is the most common presenting symptom [4]. Other presenting features being vomiting, papilloedema, focal deficits, convulsion, meningism and altered sensorium. This child presented with fever and excessive sleepiness. Fundus

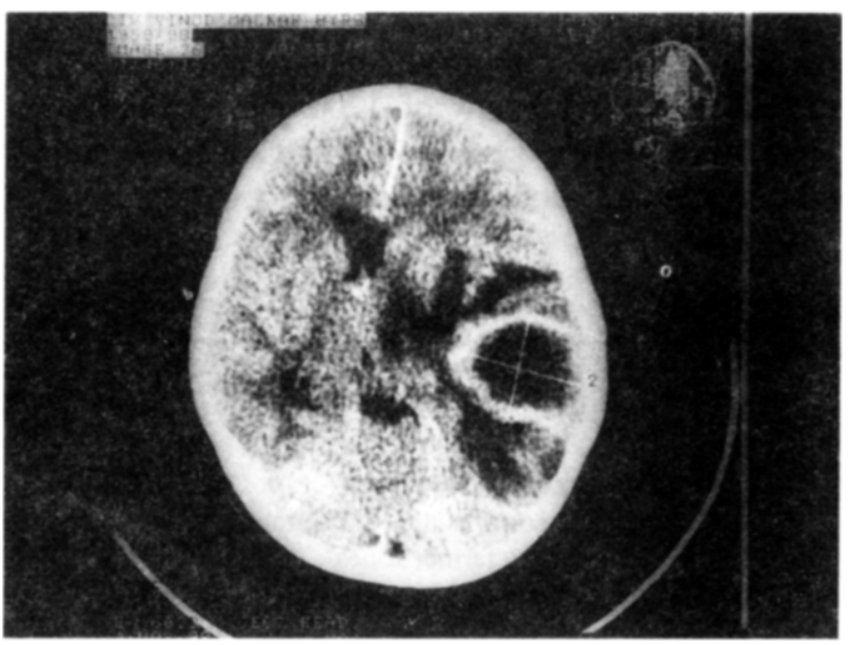

Fig. 1: Hypodense ring enhancing lesion in temporo parietal region

\footnotetext{
"Reader, Department of Paediatrics, Armed Forces Medical College, Punc - 411 (40. ${ }^{+}$Ex-Head. Department of Paediatrics, Armed Forces Medical College, Pune - $411040,{ }^{* *}$ Senior Adviser (Paediatrics). Command Iospital, (Southern Command). Pune.411 (240).
} 
was normal. There was no neurological deficit and no history of convulsion. The classical triad of headache, focal neurological deficits and fever was missing. In temporal lobe abscess, the sign earliest to appear, but often not looked for, is a homonymous hemi-anopia commencing in the superior quadrant. This is easily detected in conscious cooperative patients, but is more difficult to demonstrate when the patients are drowsy. Symptoms are present for less than a week in more than $50 \%$ of patients and for less than 2 weeks in $75 \%$ cases [6].

Rapid onset and progression is the hallmark of brain abscess. This case had only drowsiness, and within 6 hours of hospitalization developed hypertension and bradycardia. This emphasizes the importance of careful and frequent clinical monitoring in children.

A brain abscess causes an expanding intra-cranial mass effect due to cerebritis. Hence, LP should be approached cautiously and patients with brain abscess have been noted to die shortly after LP [7]. In a known case of brain abscess, LP is not only hazardous but pointless also. In this case, there was no pointer to cerebral abscess, hence, LP was attempled and no more than a few drops could be collected. Urgent CT scan clinched the diagnosis. MRI and CT are appropriate and definitive tests during the stage of cerebritis. Magnetic resonance spectroscopy may be useful in differentiation of an abscess from a neoplasm [8].

Majority of brain abscess arise by direct spread from para nasal sinuses, middle ear or mastoid infections [9]. The more common primary sites of infections include skin pustules, dental or tonsillar abscess, chronic pulmonary infections, septicemia and bacterial endocarditis. The :ite is unknown in 20 to $37 \%$ of patients [9]. In a review of 1200 cases of brain abscess, $40 \%$ were otogenic in origin. Our case had dental caries but no odontogenous cyst.
Steroids should be used in the treatment of brain abscess to help reduce cerebral edema and so an altered level of consciousness. Aspiration and excision are perfect mode of treatment. The former can be performed at any stage of brain abscess and excision is advised when the abscess is encapsulated [9].

Because of difficulty in diagnosis and management of brain abscess, mortality is high and sequelae are frequent [7]. Serial imaging will definitely reduce the morbidity and morality. Chronic seizure disorders are common after brain abscess.

\section{References}

1. Grigoriadis E, Gold WL. Pyogenic brain abscess caused by Streptococcus pneumonae : case report and review. Clin Infect Dis 1997;25:1108-12.

2. Sambasivan M. Ramamurthy B. Pyogenic Infections. In : Ramamurthy B, Tandon PN. A Text Book of Neurosurgery, $2^{\text {nd }}$ ed. BI Churchill Livingstone Pvt Ltd, New Delhi. 1996:447-67.

3. Unfulfilled expectations in cerebral abscess (editorial). BMJ 1969;21-6.

4. Bhatia R, Kak VK, Sambasivan M. Pyogenic brain abscessreview of over 1200 cases from three centers in India, $9^{\text {th }}$ International Congress Neurol Surgery, New Delhi. (Abstract) 1989;544:400-10.

5. Anderson M. Management of cerebral infections. J Neurol Neurosurg Psychiatr 1993;56:1243-58.

6. Garfield J. Management of supratentorial intracranial abscess. A review of 200 cases. BMJ 1969;2:7-11.

7. Saez-Llorens XJ, Umana MA, Odio CM. McCracken GH Jr, Nelson JD. Brain abscess in infants and children. Paediatr Infect Dis J 1989;8:449-58.

8. Martinez-Perez I, Moreno A, Alonso J, Aguas J, Conesa G, Capdevila A, et al. Diagnosis of brain abscess by magnetic resonance spectroscopy:report of two cases. J Neurosurg 1997;86:708-13.

9. Chaudhary AR, Taylor JC, Whitaker R. Primary excision of brain abscess. BMJ 1977:2:1119-21. 